\title{
Exploration of Antioxidant, Antibacterial Activities of Green Synthesized Hesperidin Loaded PLGA Nanoparticles
}

\author{
Krithiga Balakrishnan ${ }^{1,2}{ }^{(D)}$, Sangeetha C Casimeer ${ }^{3}{ }^{(D)}$, Alaa Yousef Ghidan ${ }^{4}$ (D), \\ Tawfiq M. Al Antary 5 (D), Anbu Singaravelu 1,*iD
}

1 Department of Biochemistry, Sacred Heart College (Autonomous), Tirupattur-635 601, Tirupattur, Tamil Nadu, India.

2 Department of Biochemistry, Thiruvalluvar Arts and Science College, Kurinjipadi-607302, Cuddalore, Tamil Nadu, India; biochemkrithi@gmail.com (K.B.);

3 Department of Physics, Sri Padmavati Mahila Visvavidyalayam (Women's University), Tirupati, Andhra Pradesh 517502, India; carosangee@gmail.com (C.C.S.);

4 Nanotechnology and Microbiology, Research and Development Center, The Higher Council for Science and Technology, Amman, 11941, Jordan; alaayghidan@gmail.com (A.Y.G.);

5 Department of Plant Protection, Faculty of Agriculture, University of Jordan, Amman, 11942, Jordan; tawfiqalantary@yahoo.com (T.A.A.);

* Correspondence: scanbu@gmail.com;

Received: 10.02.2021; Revised: 7.03.2021; Accepted: 9.03.2021; Published: 23.03.2021

\begin{abstract}
To elaborate on the potential benefits of hesperidin loaded PLGA nanoparticles (HES-PLGA NPs), present investigation reports about the antioxidant, antimicrobial, and cytotoxic potential of hesperidin loaded PLGA nanoparticles (HES-PLGA-NPs). The antioxidant potential of Hesperidin and HES-PLGA-NPs was demonstrated by ATBS, DPPH, Hydroxy, Hydrogen peroxide, and superoxide radical showing the dose-dependent antioxidants activity. Both the test gained better potential with HES loaded PLGA nanoparticles than free HES. HES-PLGA-NPs also proved its antibacterial activity against tested bacterial strains, including Escherichia coli, Klebsiella pneumoniae, Enterobacter aerogenes, and Pseudomonas aeruginosa. HES-PLGA-NPs had a good ability to minimize biofilms' formation and a good to weak ability to degrade pre-formed biofilms. Thus, the bionanotherapy, which has been attempted using HES-PLGA-NPs would provide a promising scope as a potent bio compound that can be replaced for medical ailments in a cost-effective, greener synthesis.
\end{abstract}

Keywords: Hesperidin; antioxidants; antibacterial; nanomedicine.

(C) 2021 by the authors. This article is an open-access article distributed under the terms and conditions of the Creative Commons Attribution (CC BY) license (https://creativecommons.org/licenses/by/4.0/).

\section{Introduction}

The exposure of antibiotic misusages and over usages contribute to antimicrobial resistance causing a major health threat in this century [1]. Martinez et al. (2015) state that a strain is resistant to antibiotic dose beyond the limit of Minimal Inhibitory Concentration [2]. Eventually, bacteria have developed resistance against many antibiotic groups, thereby creating social hazards. This uprising issue has been concerned by the World Health Organization (WHO) and taken as an alarming issue faced by the medical science, which in turn adventuring new agent against pathogen has been raised. It has focused on the natural products derived from plants, and bio components are being appreciable [3].

Phytochemicals are the secondary metabolites, a non-nutritive compound derived from plants and some edible microalgae and fungal groups. Many studies have demonstrated the 
potential effects of various phytochemicals classes in treating human diseases [4]. Antioxidant phytochemicals are the naturally occurring plant resources used in the ailment of many chronic disease pathogenesis $[5,6]$. Oxidative stress is an abnormal state formed when the antioxidant defense system fails in a cell where excess reactive oxygen species (ROS) or reactive nitrogen species (RNS) are produced, which is the major causative product for many diseases [6]. The molecule with unpaired electrons seeks to pair electrons to attain stability by quenching electrons from another molecule in a chain-like manner, thereby causing a group of unstable molecules called free radicals, oxidants, ROS leads to the damage of DNA, lipids, and Protein in a cell [7].

Flavonoids are a phenolic structured natural bioactive component with wide forms found pervasively in plants [8]. It was stated by many reports that flavonoids have multiple pharmacological effects and bioactivities. Citrus fruits contain significantly high flavonoid components, and citrus flavonoids are reported to possess many proven therapeutic benefits against cancer, diabetes, cardiovascular diseases, chronic degenerative diseases [9]. They have specific potential in an antioxidant, anti-inflammatory role which was considered the most common indicator for many pathogenic conditions [10]. Hesperidin (HES) is one of the bestknown citrus flavonoids with high pharmacological effects. HES belongs to the flavone glycoside, which is the basic constituent of many citrus plants. Hesperidin (3,5,7trihydroxyflavanone -7- rhamnoglucoside) conjugated to an aglycone hesperidin, bonded with rutinose. The glycoside part of HES is a disaccharide comprised of glucose and rhamnose. Thus, hesperidin is considered as $\beta-7$, rutinoside of hesperidin, it has a broad spectrum of biological activity [11, 12]. There are many proven studies reported on various pharmacological benefits includes anti-aging [13] (Stanisic et al., 2020), anti-carcinogenic [14], lipid-lowering [15], cardioprotective effects [16], hepatoprotective activities [17]. Though hesperidin has numerous health-related prosperities, it is also reported for its poor bioavailability and low water solubility [18]. Removal of rhamnose group from hesperidin could improve the bioavailability in the form of hesperitin-7-glucoside [19]. In recent times, nanomedicine has become a -appreciated platform for overcoming the barriers in biological, physical sciences. Nanostructures are being used in drug delivery as a drug carrier by encapsulating the therapeutic drug to achieve the target site more precisely [20].

In the midst of current nanomaterials, biodegradable polymers are widely used in the field of drug delivery. PLGA (Poly Lacto Co-glycolic acid) one of the best-featured biopolymers frequently preferred for drug delivery since it has easy hydrolyzing property inside the body [21]. The monomers are degraded into non-toxic elements viz., water and carbon dioxide as an end product. This shows that it has a more -friendly feature, which is more advantageous in designing a drug with safety and efficacy [22]. To make the biological potential of HES more available, PLGA encapsulation would be a prominent and efficient choice of selecting a supportive polymer. Hence, this investigation covers the potential focusing of HES on antioxidant and cytotoxic properties.

\section{Materials and Methods}

\subsection{Chemicals and nanoformulation.}

Sodium acetate, sodium carbonate, n-butanol, sodium hydroxide, acetone, ethanol, chloroform, aluminum chloride 2,2'-azinobis-(3-ethylbenzothiazoline-6-sulfonic acid) (ABTS), $\alpha, \alpha$-diphenyl- $\beta$-picrylhydrazyl (DPPH) and were purchased from Merck, Mumbai, 
India. Mannitol salt, Mueller Hinton Agar were obtained from Hi-media Ltd. All the chemicals used were of analytical grade. Hesperidin-loaded PLGA nanoparticles (POL-PLGA-NPs) were oil in water single emulsion solvent evaporation method, and its physiochemical features were confirmed by Transmission Electron Microscopy (TEM), dynamic light scattering (DLS), Fourier Transform Infrared Spectroscopy (FTIR), XRD and NMR analysis [23].

\subsection{DPPH assay.}

The antioxidant potential of HES/HES-PLGA-NPs was assessed by the method followed as reported by Das et al. (2013) with slight adjustments [24]. The DPPH scavenging assay is the analysis of the scavenging potential of antioxidants against stable radicals. The radical scavenging assay is resolved by UV spectrophotometer at $517 \mathrm{~nm}$. An aliquot of the fabricated HES, HES-PLGA-NPs, and Standard (Ascorbic acid) were mixed with $1 \mathrm{~mL}$ of different concentration ranging from $(12.5-100 \mu \mathrm{g} / \mathrm{mL})$ in freshly made $1 \mathrm{~mL}$ methanol DPPH solution $1 \mathrm{mM}$, followed by incubating the mixture at dark for $30 \mathrm{~min}$ and read at $517 \mathrm{nM}$. Ascorbic acid was used as the antioxidant standard. Methanol was used as a blank. IC50 is defined as the concentration $(\mu \mathrm{g} / \mathrm{mL})$ that is $50 \%$ inhibition of DPPH activity by reducing its color by the antioxidant present in the sample. It was calculated by the equation, $\operatorname{IC}_{50}(\%)=$ $100 \times\left(\mathrm{A}_{0}-\mathrm{As}\right) / \mathrm{A}_{0}$, where As and A0 are the values for the absorbance of the sample and absorbance of the negative control, respectively. Tests were performed in triplicate.

\subsection{Hydrogen peroxide scavenging assay.}

$\mathrm{H}_{2} \mathrm{O}_{2}$ radical scavenging assay was performed as reported by Arai et al. (2020) [25]. The assay is done by mixing various concentrations of HES, HES-PLGA-NPs, and Standard (Ascorbic acid) $(12.5-100 \mu \mathrm{g} / \mathrm{mL})$ to $0.6 \mathrm{~mL}$ of $\mathrm{H}_{2} \mathrm{O}_{2}$ prepared in phosphate buffer solution adjusted to $(40 \mathrm{mM}, 7.4 \mathrm{pH}) . \mathrm{H}_{2} \mathrm{O}_{2}$ alone was used as a blank. Ascorbic acid was used as a standard along with phosphate buffer $(40 \mathrm{mM}, 7.4 \mathrm{pH})$. Samples were vortexed, and the supernatant was kept incubated for $10 \mathrm{~min}$ at room temperature. The intensity was determined by UV-visible spectroscopy and read at $610 \mathrm{~nm}$. The percentage inhibition was calculated using the formula, $\mathrm{IC}_{50}(\%)=100 \times(\mathrm{Ac}-\mathrm{As}) / \mathrm{Ac}$, where As and $\mathrm{A}_{0}$ are the values for the absorbance of the sample and absorbance of the negative control, respectively. Tests were performed in triplicate.

\subsection{Hydrogen radical scavenging assay.}

Hydroxyl radical $(\bullet \mathrm{OH})$ scavenging activity was carried out as reported by Kumar et al. (2015) with some modifications [26]. $\bullet \mathrm{OH}$ radicals were performed using a usual Fenton reaction made by a fresh reaction mixture composed of $\mathrm{FeSO}_{4}$ and $\mathrm{H}_{2} \mathrm{O}_{2}$. To the mixture, 1.2 $\mathrm{mL}$ of $0.1 \mathrm{~mL}$ of phosphate buffer and the samples (HES, HES-PLGA-NPs, and Standard Ascorbic acid) at various concentrations ranging from $12.5-100 \mu \mathrm{g} / \mathrm{mL}$ were taken. $1 \mathrm{~mL}$ of $3 \%$ TCA and TBA were mixed and kept in a boiling water bath for $10 \mathrm{~min}$. Absorbance was measured at $532 \mathrm{~nm}$ after the mixture was cooled. The percentage inhibition was calculated using the formula, $\mathrm{IC}_{50}(\%)=100 \times\left(\mathrm{Ac}_{\mathrm{c}} \mathrm{As}\right) / \mathrm{Ac}$, where As and $\mathrm{A}_{0}$ are the values for the absorbance of the sample and absorbance of the negative control, respectively. Tests were performed in triplicate. 


\subsection{Superoxide radical scavenging activity}

Superoxide radical scavenging assay was performed as reported by Pool et al. (2012) [27]. It is based on the oxidation of NADH by the Phenozinemethosulphate system, which reduces Nitro blue tetrazolium to complex violet-colored formation due to the production of superoxide radicals. The reduction of color is due to the antioxidant scavenging factor of the sample. The mixture is containing phosphate buffer ( $0.1 \mathrm{M}, \mathrm{pH}-8)$, NADH (73 $\mu \mathrm{M}$, PMS (15 $\mu \mathrm{M})$, NBT $(50 \mu \mathrm{M})$ and various concentration of HES, HES-PLGA-NPs, and Standard (Ascorbic acid) in methanol $(12.5-100 \mu \mathrm{g} / \mathrm{mL})$. The mixture was incubated at ambient conditions for 5 minutes, and the intensity was recorded at $560 \mathrm{~nm}$. The percentage inhibition was calculated using the formula, $\mathrm{IC}_{50}(\%)=100 \times\left(\mathrm{Ac}_{\mathrm{c}}-\mathrm{As}\right) / \mathrm{Ac}$, where $\mathrm{A}_{\mathrm{s}}$ and $\mathrm{A}_{0}$ are the values for the absorbance of the sample and absorbance of the negative control, respectively. Tests were performed in triplicate.

\subsection{Antibacterial activity.}

To assess the antibacterial potential of HES-PLGA-NPs, a standard well diffusion method was followed. The multi-drug resistant-clinical isolates such as Escherichia coli, Klebsiella pneumoniae, and Enterobacter aerogenes, Pseudomonas aeruginosa were obtained from Sri Narayani Hospital \& Research Centre, Vellore, Tamil Nadu, India. For the purpose of getting fresh culture, the nutrient broth was prepared to sub-culture the bacteria. $50 \mu \mathrm{L}$ of stock culture was mixed with $950 \mu \mathrm{L}$ of sterile Muller Hinton broth and incubated at $37^{\circ} \mathrm{C}$ for $24 \mathrm{~h}$. From this overnight culture, $50 \mu \mathrm{L}$ of bacteria was taken and spread onto the solidified Muller Hinton agar, and wells were made accordingly [28]. The sample HES-PLGA-NPs (25, 50, 75, $100 \mu \mathrm{g} / \mathrm{mL})$, while Gentamycin $(10 \mu \mathrm{g} / \mathrm{mL})$ was used as a standard control, were added in the well. Then the plates were incubated at $37^{\circ} \mathrm{C}$ overnight. The zone of inhibition was measured in mm using a measurement scale. Also, the Biofilm inhibition activity of HES-PLGA NPs was assessed by the method described by Mariadoss et al. (2019) [29].

\section{Results and Discussion}

The result has confirmed the antioxidant potential of HES-PLGA-NPs and ascorbic acid. The antioxidants potential was assessed by documented the $\mathrm{IC}_{50}$ concentration of DPPH, ABTS, superoxide dismutase (SOD), Hydroxy radical $(\mathrm{OH})$, and Hydrogen peroxide $\left(\mathrm{H}_{2} \mathrm{O}_{2}\right)$ radical scavenging activity. The antioxidant scavenging effect of DPPH radical expressed as IC50 values were in the following order: Ascorbic acid $(24.62 \pm 2.45 \mu \mathrm{g} / \mathrm{mL})$, HES-PLGA-NPs $(81.32 \pm 0.59 \mu \mathrm{g} / \mathrm{mL}$ ), and HES was found to $88.32 \pm 3.05 \mu \mathrm{g} / \mathrm{mL}$ (Fig. 1a). While the IC50 values ABTS radical scavenging activity was in the following order: Ascorbic acid $(14.37 \pm 1.58 \mu \mathrm{g} / \mathrm{mL})$, HES-PLGA-NPs $(44.63 \pm 5.11 \mu \mathrm{g} / \mathrm{mL})$, and HES was found to $39.11 \pm 1.02$ $\mu \mathrm{g} / \mathrm{mL}$ (Fig. 1b).

Hydrogen peroxide scavenging assay confirmed that HES has an $\mathrm{IC}_{50}$ concentration of $65.45 \pm 4.01 \mu \mathrm{g} / \mathrm{mL}$, and HES-PLGA-NPs has $58.18 \pm 3.72 \mu \mathrm{g} / \mathrm{mL}$ while ascorbic acid has $22.95 \pm 2.91 \mu \mathrm{g} / \mathrm{mL}$ (Fig. 2a). It shows that the fabricated HES-PLGA-NPs have a significant antioxidant activity when compared with standard ascorbic acid. The result of the hydroxyl radical assay confirmed that HES has an IC50 concentration of $76.153 .16 \pm \mu \mathrm{g} / \mathrm{mL}$ and HESPLGA-NPs has $75.67 \pm 6.05 \mu \mathrm{g} / \mathrm{mL}$ while ascorbic acid has $19.47 \pm 4.85 \mu \mathrm{g} / \mathrm{mL}$. It shows that the fabricated HES-PLGA-NPs have more antioxidant activity than a standard ascorbic acid (Fig. $2 b)$. 

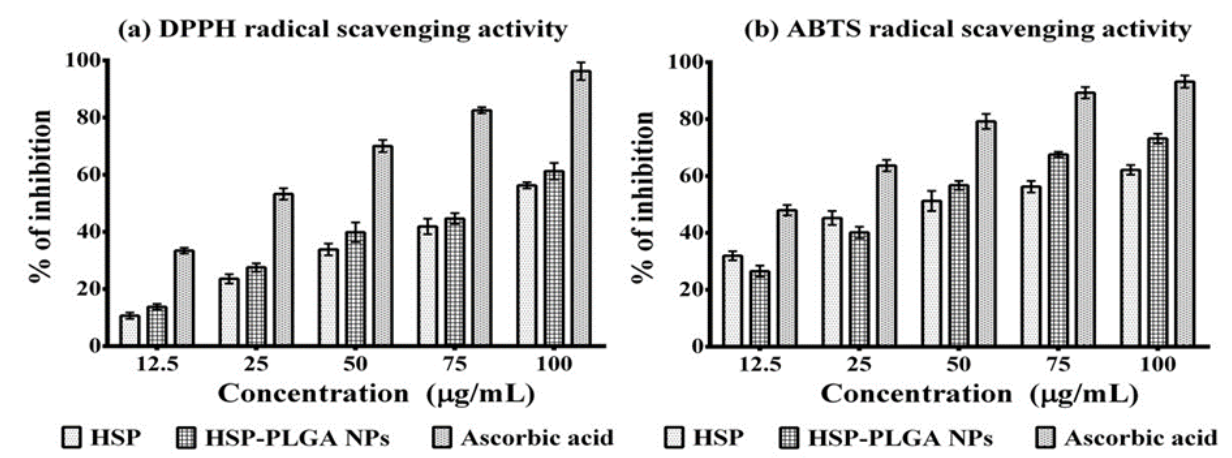

Figure 1. DPPH (a) and ABTS (b) radical scavenging activity of HES, HES-PLGA-NPs, and ascorbic acid. The values are expressed as Mean $\pm \mathrm{SD}$ of three independent experiments.
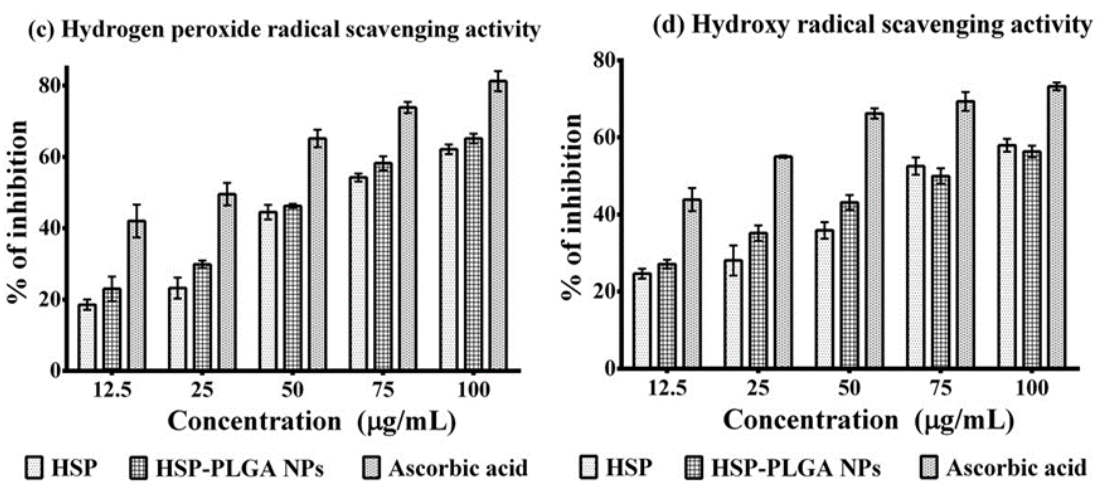

Figure 2. Hydrogen peroxide (a) and hydroxyl (b) radical scavenging activity of HES, HES-PLGA-NPs and ascorbic acid. The values are expressed as Mean \pm SD of three independent experiments.

Superoxide scavenging activity confirmed that HES has an IC50 concentration of $92.17 \pm 3.71 \mu \mathrm{g} / \mathrm{mL}$ and HES-PLGA-NPs has $81.56 \pm 4.09 \mu \mathrm{g} / \mathrm{mL}$ while ascorbic acid has $20.05 \pm 5.94 \mu \mathrm{g} / \mathrm{mL}$. It shows that the fabricated HES-PLGA-NPs have more antioxidant activity than a standard ascorbic acid (Fig. 3).

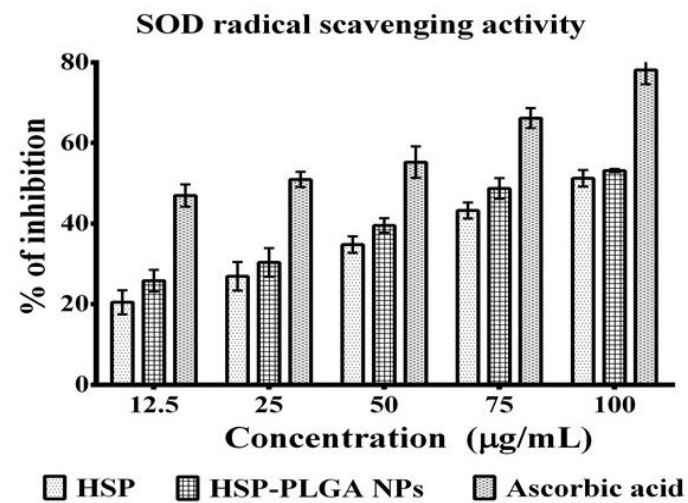

Figure 3. Superoxide radical scavenging activity of HES, HES-PLGA-NPs, and ascorbic acid. The values are expressed as Mean \pm SD of three independent experiments.

By observing the results of DPPH, ABTS, SOD, $\mathrm{OH}$, and $\mathrm{H}_{2} \mathrm{O}_{2}$ radical scavenging assay, it clearly shows that the fabricated HES-PLGA-NPs have high antioxidant potential; thus, it quenched the stable free radicals in the reaction. It was reported well in a study made by De Oliveira et al. (2013) that HES can modulate the antioxidant circumstances [30]. They studied the rat of continuous swimming group and interval swimming group on a note of exercise protocols and related oxidative disorders; it showed that HES supplementation has 
improved the antioxidant biomarkers and lowered lipidemic effects. Hence, to improve the bioavailability of HES, PLGA encapsulated NPs would contribute more antioxidant profile in the conditions of oxidative damages.

This was planned to use synthesized HES-PLGA-NPs as a substitute for conventional antibiotics in the fight against intracellular pathogenic bacteria, particularly drug-resistant bacterial strains including Escherichia coli, Klebsiella pneumoniae, Enterobacter aerogenes, and Pseudomonas aeruginosa. The antimicrobial activity of HES-PLGA-NPs against bacterial strain was seen in figure 4.

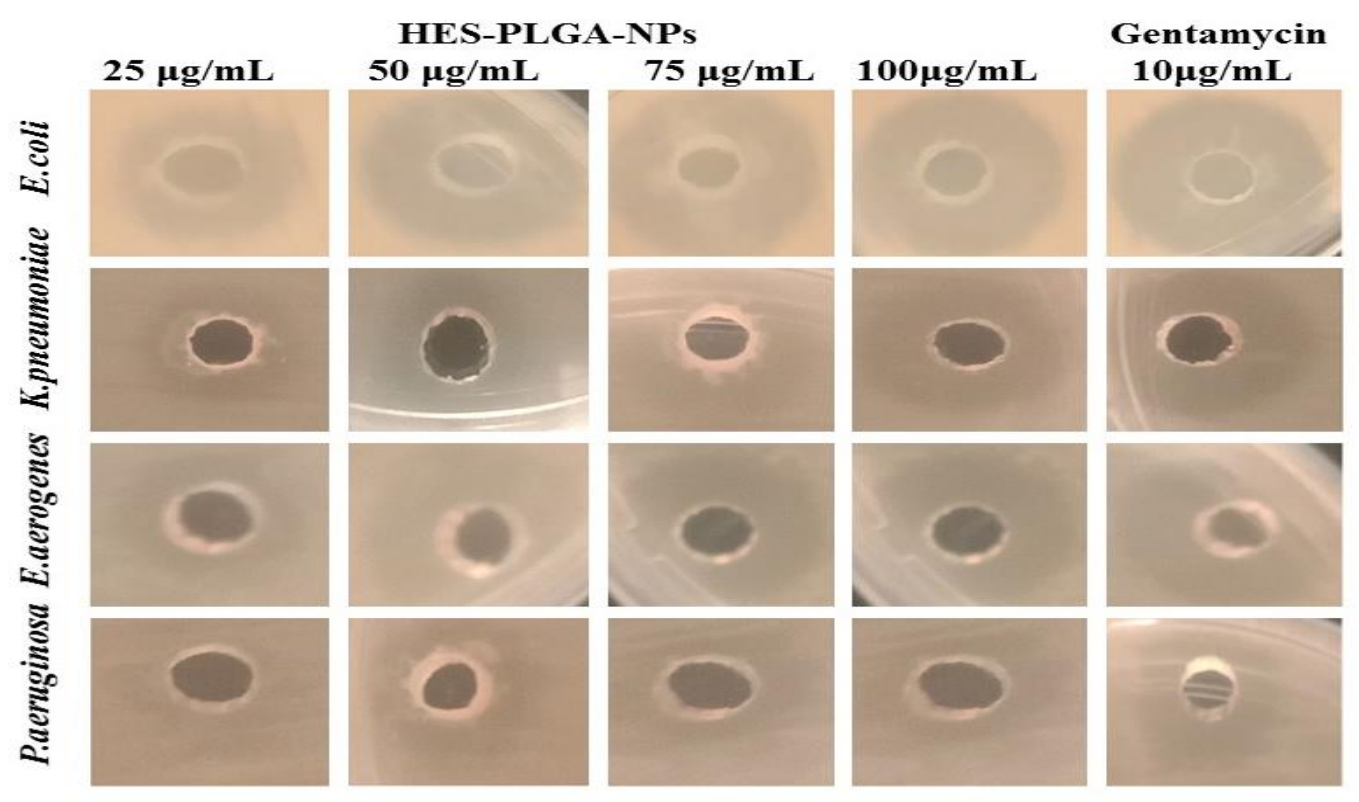

Figure 4. Growth inhibition activity of HES-PLGA-NPs screened by well diffusion method.

Table 1. Antimicrobial screening test of different concentrations of HES-PLGA-NPs against some bacterial

\begin{tabular}{lccccc}
\multicolumn{7}{c}{ strains. } \\
\hline Microorganism & $\mathbf{2 5} \mathbf{\mu g} / \mathbf{m L}$ & $\mathbf{5 0} \mathbf{\mu g} / \mathbf{m L}$ & $\mathbf{7 5} \boldsymbol{\mu \mathbf { g }} / \mathbf{m L}$ & $\mathbf{1 0 0} \boldsymbol{\mu \mathbf { g } / \mathbf { m L }}$ & $\begin{array}{c}\text { Gentamycin }(\mathbf{1 0} \\
\end{array}$ \\
\hline E.coli & $10.8 \pm 0.91$ & $13.4 \pm 0.32$ & $16.1 \pm 0.40$ & $23.8 \pm 0.50$ & $28.3 \pm 0.20$ \\
K.pneumoniae & $7.6 \pm 0.24$ & $9.7 \pm 0.45$ & $13.5 \pm 0.30$ & $21.6 \pm 0.40$ & $27.5 \pm 0.10$ \\
E.aerogenes & $5.7 \pm 0.10$ & $8.5 \pm 0.10$ & $16.1 \pm 0.20$ & $22.4 \pm 0.20$ & $25.3 \pm 0.30$ \\
P.aeruginosa & $10.3 \pm 0.35$ & $11.6 \pm 0.32$ & $16.6 \pm 0.10$ & $23.6 \pm 0.10$ & $27.5 \pm 0.40$ \\
\hline
\end{tabular}

As shown in Table 1, the antimicrobial actions of the HES-PLGA-NPs were increased with an increase in the concentration of HES-PLGA-NPs compared with the standard drug. The antimicrobial sensitivity results have shown that the highest zone of inhibition was measured in E.coli $(23.8 \pm 0.50 \mathrm{~nm})$, K.pneumoniae $(21.6 \pm 0.40 \mathrm{~nm})$, E.aerogenes $(18.4 \pm 0.20 \mathrm{~nm})$, and P.aeruginosa $(23.6 \pm 0.10 \mathrm{~nm})$ at $100 \mu \mathrm{g} / \mathrm{mL}$, which indicates that HESPLGA-NPs has a broad-spectrum antibiotic potential to act against both gram-positive and gram-negative bacteria.

The zone of inhibition demonstrates that HES-PLGA-NPs have higher antimicrobial activity against E.coli. It shows a concentration dependence antibacterial activity. Also, the biofilm inhibition activity of HES-PLGS NPs against Pseudomonas aeruginosa, Klebsiella pneumoniae, Enterobacter aerogenes, and Escherichia coli are as follows 38.25\%, 42.23\%, $67.54 \%$, and $54.59 \%$ (Fig. 5). The biofilm inhibition assay shows that HES-PLGS NPs have bacteriostatic behavior while the highest concentration reveals clear bactericidal activity. The concentrations found were comparable to the results of Karayıldırım et al., (2017) that HES is 
the alternative choice of an antibacterial agent as a novel therapy in the approach drug delivery [31].

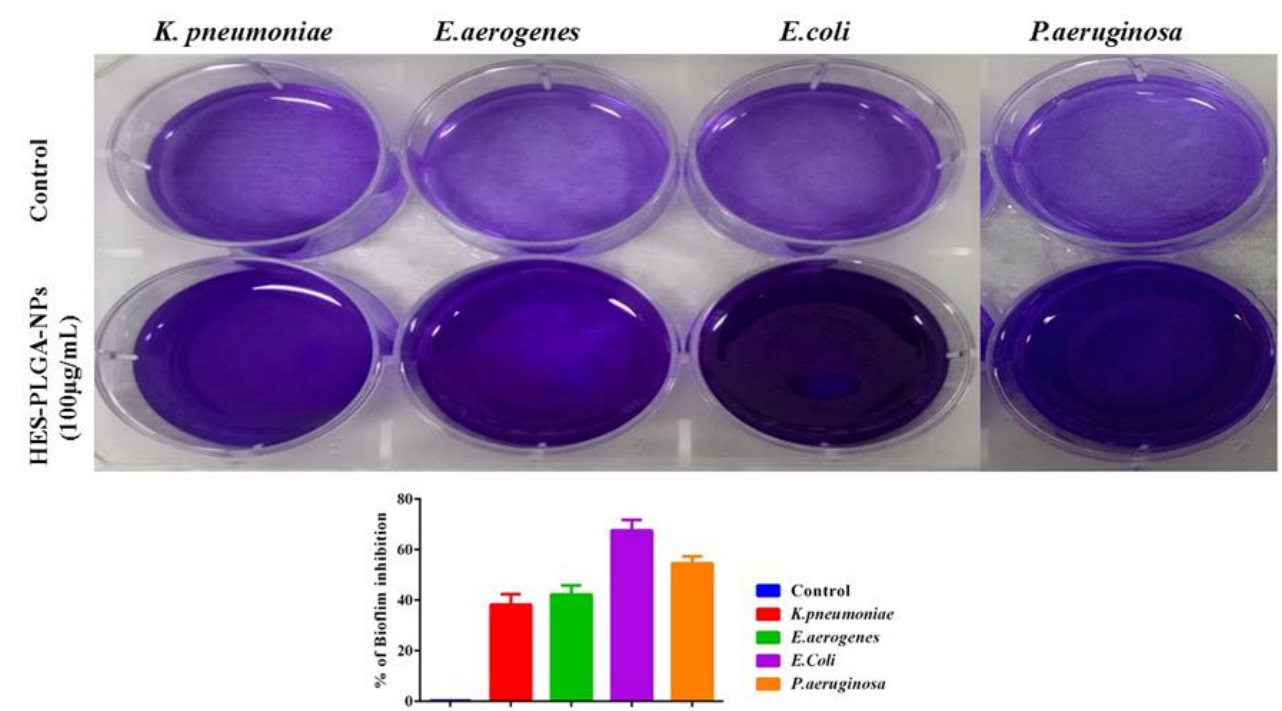

Figure 5. Bioflim inhibition activity of HES-PLGA-NPs screened by using crystal violet staining method.

\section{Conclusions}

Attending this investigation reveals an effect of HES-PLGA-NPs on antioxidant and antimicrobial profiles. The results showed the evident benefits of HES-PLGA NPs, which could be taken for various pathophysiological conditions upon bacterial diseases and oxidative stress-related conditions. The discovery of this fabricated bio polymer-based phytonanotherapy has a great pipeline in finding medications against antibacterial resistant factors and antioxidant defenses.

\section{Funding}

This research received no external funding.

\section{Acknowledgments}

The authors are thankful to Sacred Heart College (Autonomous) authorities, Tirupattur-635 601, Tirupattur, Tamil Nadu, India, for providing the necessary support to carry out this research work.

\section{Conflicts of Interest}

The authors declare no conflict of interest.

\section{References}

1. Rusic, D.; Bukic, J.; Seselja Perisin, A.; Leskur, D.; Modun, D.; Petric, A.; Vilovic, M.; Bozic, J. Are We Making the Most of Community Pharmacies? Implementation of Antimicrobial Stewardship Measures in Community Pharmacies. A Narrative Review. Antibiotics 2021, 10, https://doi.org/10.3390/antibiotics10010063.

2. Martinez, J.L.; Coque, T.M. Baquero F. What is a resistance gene? Ranking risk in resistomes. Nat Rev Microbiol 2015, 13, 116-23, https://doi.org/10.1038/nrmicro3399.

3. Cheesman, M.J.; Ilanko, A.; Blonk, B.; Cock, I.E. Developing new antimicrobial therapies: are synergistic combinations of plant extracts/compounds with conventional antibiotics the solution? Pharmacognosy Reviews 2017, 11, https://doi.org/10.4103/phrev.phrev_21_17. 
4. Mariadoss, A.V.; Vinyagam, R.; Rajamanickam, V.; Sankaran, V.; Venkatesan, S.; David, E. Pharmacological aspects and potential use of phloretin: A systemic review. Mini Reviews in Medicinal Chemistry 2019, 19, 1060-7, https://doi.org/10.2174/1389557519666190311154425.

5. Mariadoss, A.V.; Vinayagam, R.; Xu, B.; Venkatachalam, K.; Sankaran,V.; Vijayakumar, S.; Bakthavatsalam, S.R.; Mohamed, S.A.; David, E. Phloretin loaded chitosan nanoparticles enhance the antioxidants and apoptotic mechanisms in DMBA induced experimental carcinogenesis. ChemicoBiological Interactions 2019, 1, 308, 11-9, https://doi.org/10.1016/j.cbi.2019.05.008.

6. Venkatachalam, K.; Vinayagam, R.; Arokia Vijaya Anand, M.; Isa, N.M.; Ponnaiyan, R. Biochemical and molecular aspects of 1,2-dimethylhydrazine (DMH)-induced colon carcinogenesis: a review. Toxicology Research 2020, 9, 2-18, https://doi.org/10.1093/toxres/tfaa004.

7. Atta, E.M.; Mohamed, N.H.; Abdelgawad, A.A. Antioxidants: An overview on the natural and synthetic types. European Chemical Bulletin 2016, 6, 365-75, http://dx.doi.org/10.17628/ecb.2017.6.365-375.

8. Ali ,S.S.; Ahsan, H.; Zia, M.K.; Siddiqui, T.; Khan, F.H. Understanding oxidants and antioxidants: classical team with new players. Journal of food biochemistry 2020, 44, https://doi.org/10.1111/jfbc.13145.

9. Rafiq, S.; Kaul, R.; Sofi, S.A.; Bashir, N.; Nazir, F.; Nayik, G.A. Citrus peel as a source of functional ingredient: A review. Journal of the Saudi Society of Agricultural Sciences 2018, 17, 351-8, https://doi.org/10.1016/j.jssas.2016.07.006.

10. Yi, L.; Ma, S.; Ren, D. Phytochemistry and bioactivity of Citrus flavonoids: a focus on antioxidant, antiinflammatory, anticancer and cardiovascular protection activities. Phytochemistry Reviews 2017, 16, 479511, https://doi.org/10.1007/s11101-017-9497-1.

11. Mahmoud, A.M.; Hernandez Bautista, R.J.; Sandhu, M.A.; Hussein, O.E. Beneficial effects of citrus flavonoids on cardiovascular and metabolic health. Oxidative Medicine and Cellular Longevity 2019, 2019, https://doi.org/10.1155/2019/5484138.

12. Srinivasan, S.; Vinothkumar, V.; Murali, R. Antidiabetic Efficacy of Citrus Fruits with Special Allusion to Flavone Glycosides. Bioactive Food as Dietary Interventions for Diabetes 2019, 335-346, https://doi.org/10.1016/B978-0-12-813822-9.00022-9.

13. Stanisic, D.; Liu, L.H.; Dos Santos, R.V.; Costa, AF.; Durán, N.; Tasic, L. New Sustainable Process for Hesperidin Isolation and Anti-Ageing Effects of Hesperidin Nanocrystals. Molecules 2020, 25, https://doi.org/10.3390/molecules25194534.

14. Park, Y.B.; Do, K.M.; Bok, S.H.; Lee, M.K.; Jeong, T.S.; Choi, M.S. Interactive effect of hesperidin and vitamin E supplements on cholesterol metabolism in high cholesterol-fed rats. International Journal for Vitamin and Nutrition Research 2001, 71, 36-44, https://doi.org/10.1024/0300-9831.71.1.36.

15. Aggarwal, V.; Tuli, H.S.; Thakral, F.; Singhal, P.; Aggarwal, D.; Srivastava, S.; Pandey, A.; Sak, K.; Varol, M.; Khan, M.A.; Sethi, G. Molecular mechanisms of action of hesperidin in cancer: Recent trends and advancements. Experimental Biology and Medicine 2020, 245, 486-97, https://doi.org/10.1177/1535370220903671.

16. Rezaee, R.; Sheidary, A.; Jangjoo, S.; Ekhtiary, S.; Bagheri, S.; Kohkan, Z.; Dadres, M.; Oana Docea, A.; Tsarouhas, K.; Sarigiannis, D.A.; Karakitsios, S. Cardioprotective effects of hesperidin on carbon monoxide poisoned in rats. Drug and Chemical Toxicology 2019, 1-6, https://doi.org/10.1080/01480545.2019.1650753.

17. Zaghloul, R.A.; Elsherbiny, N.M.; Kenawy, H.I.; El-Karef, A.; Eissa, L.A.; El-Shishtawy, M.M. Hepatoprotective effect of hesperidin in hepatocellular carcinoma: Involvement of Wnt signaling pathways. Life Sciences 2017, 185, 114-25, https://doi.org/10.1016/j.lfs.2017.07.026.

18. Li, Y.M.; Li, X.M.; Li, G.M.; Du, W.C.; Zhang, J.; Li, W.X.; Xu, J.; Hu, M.; Zhu, Z. In vivo Pharmacokinetics of Hesperidin are affected by treatment with glucosidase-like BglA protein isolated from yeasts. Journal of Agricultural and Food Chemistry 2008, 56, 5550-7, https://doi.org/10.1021/jf800105c.

19. Habauzit, V.; Nielsen, I.L.; Gil-Izquierdo, A.; Trzeciakiewicz, A.; Morand, C.; Chee, W.; Barron, D.; Lebecque, P.; Davicco, M.J.; Williamson, G.; Offord, E. Increased bioavailability of hesperetin-7-glucoside compared with hesperidin results in more efficient prevention of bone loss in adult ovariectomised rats. British Journal of Nutrition 2009, 102, 976-84, https://doi.org/10.1017/S0007114509338830.

20. Patra, J.K.; Das, G.; Fraceto, L.F.; Campos, E.V.; del Pilar Rodriguez-Torres, M.; Acosta-Torres, L.S.; DiazTorres, L.A.; Grillo, R.; Swamy, M.K.; Sharma, S.; Habtemariam, S. Nano based drug delivery systems: recent developments and future prospects. Journal of Nanobiotechnology 2018, 16, https://doi.org/10.1186/s12951-018-0392-8.

21. Su, S.; Kang, P.M. Systemic Review of Biodegradable Nanomaterials in Nanomedicine. Nanomaterials 2020, 10, https://doi.org/10.3390/nano10040656.

22. Washington, M.A.; Balmert, S.C.; Fedorchak, M.V.; Little, S.R.; Watkins, S.C.; Meyer, T.Y. Monomer sequence in PLGA microparticles: Effects on acidic microclimates and in vivo inflammatory response. Acta Biomaterialia 2018, 65, 259-71, https://doi.org/10.1016/j.actbio.2017.10.043.

23. Krithiga, B.; Casimeer, S.C.; Ghidan, A.Y.; Ghethan FY, Venkatachalam K, Singaravelu A. Bioformulated hesperidin-loaded PLGA nanoparticles counteract the mitochondrial-mediated intrinsic apoptotic pathway in cancer cells. Journal of Inorganic and Organometallic Polymers and Materials 2020, 31, 331-343, https://doi.org/10.1007/s10904-020-01746-9. 
24. Das, D.; Nath, B.C.; Phukon, P.; Dolui, S.K. Synthesis of ZnO nanoparticles and evaluation of antioxidant and cytotoxic activity. Colloids and Surfaces B: Biointerfaces 2013, 111, 556-60, https://doi.org/10.1016/j.colsurfb.2013.06.041.

25. Arai, Y.; Park, H.; Park, S.; Kim, D.; Baek, I.; Jeong, L.; Kim, B.J.; Park, K.; Lee, D.; Lee, S.H. Bile acidbased dual-functional prodrug nanoparticles for bone regeneration through hydrogen peroxide scavenging and osteogenic differentiation of mesenchymal stem cells. Journal of Controlled Release 2020, 328, 596607, https://doi.org/10.1016/j.jconrel.2020.09.023.

26. Kumar, S.P.; Birundha, K.; Kaveri, K.; Devi, K.R. Antioxidant studies of chitosan nanoparticles containing naringenin and their cytotoxicity effects in lung cancer cells. International Journal of Biological Macromolecules 2015, 78, 87-95, https://doi.org/10.1016/j.ijbiomac.2015.03.045.

27. Pool, H.; Quintanar, D.; Figueroa, J.D.; Marinho Mano, C.; Bechara, J.E.; Godínez, L.A.; Mendoza, S. Antioxidant effects of quercetin and catechin encapsulated into PLGA nanoparticles. Journal of Nanomaterials 2012, 2012, https://doi.org/10.1155/2012/145380.

28. Mariadoss, A.V.; Ramachandran, V.; Shalini, V.; Agilan ,B.; Franklin, J.H.; Sanjay, K.; Alaa, Y.G.; Tawfiq, M.A.; Ernest, D. Green synthesis, characterization and antibacterial activity of silver nanoparticles by Malus domestica and its cytotoxic effect on (MCF-7) cell line. Microbial Pathogenesis 2019, 135, https://doi.org/10.1016/j.micpath.2019.103609.

29. Gao, Y.; Arokia Vijaya Anand, M.; Ramachandran, V.; Venkatachalam, K.; Shalini, V.; Sankaran,V.; David, E. Biofabrication of Zinc Oxide Nanoparticles from Aspergillus niger, Their Antioxidant, Antimicrobial and Anticancer Activity. Journal of Cluster Science 2019, 30, 937-946, https://doi.org/10.1007/s10876-01901551-6.

30. de Oliveira, D.M.; Dourado, G.K.; Cesar, T.B. Hesperidin associated with continuous and interval swimming improved biochemical and oxidative biomarkers in rats. Journal of the International Society of Sports Nutrition 2013, 10, https://doi.org/10.1186/1550-2783-10-27.

31. Karayıldırım, Ç.K. Characterization and in vitro evolution of antibacterial efficacy of novel hesperidin microemulsion. Celal Bayar Üniversitesi Fen Bilimleri Dergisi 2017, 13, 943-7. 